\title{
Study on Clinical Efficacy of Chinese Medicine for No Nerve Injury Thoracolumbar Vertebral Compression Fracture Kyphosis
}

\author{
Xuzhao $\mathrm{Du}^{1, \mathrm{a}}$, Suling Deng ${ }^{1, b_{*}}$, Dongliang Shi, c, Qingliang Meng ${ }^{1, d}$, Huimin \\ $\mathrm{Gu}^{1, \mathrm{e}}$, Hao Yang ${ }^{1, \mathrm{f}_{*}}$ \\ ${ }^{1}$ Henan Province Hospital of Traditional Chinese Medicine, Zhengzhou, Henan, 450002 \\ ${ }^{\mathrm{a}}$ email, ${ }^{\mathrm{b} *}$ email, ${ }^{\mathrm{c}}$ email, ${ }^{\mathrm{d}}$ email, ${ }^{\mathrm{e}}$ email, ${ }^{\mathrm{f}}$ email,
}

Keywords: Chinese Medicine; No Nerve Injury Thoracolumbar Vertebral Compression Fractures; Convex Deformity

\begin{abstract}
Objective: To observe and analyze Chinese medicine therapy without nerve injury thoracolumbar compression fracture kyphosis clinical efficacy. Methods; The study of December 2012 - between June 2014 in our hospital for treatment of non-nerve injury thoracolumbar vertebral compression fractures were 80 cases, according to the different treatment methods will be divided into a control group ( $n=40$ patients) and the observation group ( $n=40$ patients), the observation group were treated with combined therapy of traditional Chinese medicine, Chinese medicine and western medicine control group and exercise therapy, two groups were compared using the incidence of delayed symptoms of different treatment methods and treatment changes in pain scores and spinal kyphosis Cobb's angle. Results; observation group after patients and control patients kyphosis Cobb's angle than treatment significantly improved $\mathrm{P}<0.05$; observation group kyphosis Cobb's angle was significantly less than the control group, the difference was significant $\mathrm{P}<0.05$; Observation 2 year follow-up group kyphosis Cobb's angle was significantly less than the control group, the difference was significant $\mathrm{P}<0.05$. After the observation group and the control group of patients pain patients before treatment were significantly alleviated, $\mathrm{P}<0.05$;; pain relief observation group than the control group, the difference was significant $\mathrm{P}<0.05$. Conclusion; no nerve injury thoracolumbar vertebral compression fractures were treated with combined therapy of traditional Chinese medicine, can effectively relieve the injured speedy pain state is conducive to correct vertebral kyphosis and effective restoration of vertebral height, a significant effect.
\end{abstract}

\section{Introduction}

Thoracolumbar compression fractures spine surgery is one of the more common diseases in clinical fracture, thoracolumbar fractures higher incidence of about $68 \%$, of which the vast majority of patients with vertebral compression fractures are not associated with injury symptoms . In the clinical treatment of non-nerve injury thoracolumbar vertebral compression fracture patients were treated conservatively. The study of traditional Chinese medicine and Western medicine treatment of comprehensive treatment and no nerve injury thoracolumbar vertebral compression fracture patients, comparing the efficacy of two treatment methods, are summarized below.

\section{Subjects and Methods}

The study of December 2012 - between June 2014 in our hospital for treatment of non-nerve injury thoracolumbar vertebral compression fractures of 80 patients, all subjects are in line with the relevant literature no nerve injury thoracolumbar vertebral compression diagnose fractures standards; that is, patient trauma, have a more significant lower back pain, the pain was aggravated by hand pressure, accompanied by symptoms of kyphosis, lumbar dysfunction exists; after Imaging CT scan showed that trabecular bone disorder and bone fracture, after radiographic X-ray showed bone discontinuity and the wedge-like changes. Exclude younger than 18 years old, more than 70-year-old patient; excluded patients with kyphosis angle greater than $35^{\circ}$ while spinal stenosis greater than $35 \%$ of patients and is not associated with nerve damage. Depending on the method of 
treatment will be divided into a control group ( $n=40$ patients) and observation group ( $n=40$ cases) and control group patients, 13 females, 27 males; the minimum age is 19 years old and the oldest was 66 years old median age $(40.4 \pm 2.1)$ years;

The type of injury; 5 cases of falls, 14 cases of high-altitude fall, 21 cases of traffic accidents; observation group patients, 15 females, 25 males; the minimum age is 20 years old and the oldest was 68 years, median age $(42.4 \pm 2.8$ ) years; the type of injury; six cases of falls, 14 cases of high-altitude fall, 20 cases of traffic accidents; the type of injury all subjects, age, sex and other baseline data, using SPSS20.0 statistical software package for data processing, the results showed no statistically significant, comparable, $\mathrm{p}>0.05$.

Observation group were treated with combined therapy of traditional Chinese medicine, treatment methods are as follows; (1) first be administered to a patient diagnosis and treatment of oral medicine, prescription: 15g Epimedium, 12g psoralen, $10 \mathrm{~g}$ walnuts, $10 \mathrm{~g}$ safflower, $10 \mathrm{~g}$ Eucommia, $12 \mathrm{~g}$ teasel, Wulingzhi $10 \mathrm{~g}, 10 \mathrm{~g}$ cattle Teng, $10 \mathrm{~g}$ angelica, $10 \mathrm{~g}$ red peony, $10 \mathrm{~g}$ Salvia, 12g Chuanxiong, 10g Drynaria. Patients in the first 15 days after the injury, in addition to the basic prescription Rehmanniae 30g, 10g Eupolyphaga, 15g TCS, Bupleurum 6g, 10g woody, 12g Poria, $10 \mathrm{~g}$ rhubarb; the medium-term in the above basic prescription plus Schisandra $6 \mathrm{~g}, 30 \mathrm{~g}$ astragalus; late in the above basic living alone prescription plus 10g, $10 \mathrm{~g}$ Gentiana, Clematis 10g; water torment, one day, sooner or later, each serving a second. (2) pillow repose; injured patients just need a few days before Woyu hard board bed, supine position from the first five days after the injury began with a soft pillow on the side of the vertebral body parts injured injured the initial height of the patient can withstand limited degree, a gradual increase in height, to be added to the height of the pillow $150 \sim 20 \mathrm{~cm}$. (3) give injured TDP irradiation and topical medicine, the pain in our hospital homemade paste, affixed to the injured site of back pain, one day, while using lamp, irradiation for 20 minutes a day, twice a day for treatment 2 day. (4) functional exercise guidance; 72 hours after admission, guide the injured lower back muscle exercise, 5-point method; 15 days to 3-point, slowly over the bridge-type support method, remember; not excessive exercise, in patients with limited tolerance, gradual effectively increase the intensity and frequency.

The control group with Western and Chinese medicine and exercise therapy, treatment is as follows; administered to a patient injury jiegu tablet (Zhunzi Z21021461 pharmaceutical companies in Dalian Metro Co., Ltd.), 3 times a day, every four, for taking a month ; intramuscularly administered to a patient bone peptide injection (Zhunzi H20051236 Medisan Pharmaceutical Co., Ltd.) at a dose of $4 \mathrm{mI}$ added at a concentration of 5\% glucose injection 250mI, 1 times a day, intravenous drip, continuous treatment for 15 days; function exercise as treatment and observation groups. All patients with absolute bed rest for 90 days, get out of bed must be effectively protected activities under the brace, while 90 days is absolutely prohibited load.

Patients were followed up for 2 years, using the VAS score, eye detection in patients with back pain cases; kyphosis measurements, the patient into the hospital when you get out of bed at 2 years of follow-up and well, are using imaging X-ray examination, after measurement Cobb's projection angle.

The collected set of experimental data, statistical software for data processing in data package SPSS20.0, count data and measurement data were applied (n\%) and ( $\square \mathrm{x} \pm \mathrm{s}$ ) that the differences between the groups by $\mathrm{x}^{2}$ and t-test, when $\mathrm{P}<0.05$, the difference was statistically significant.

\section{Result}

Observation group after patients and control patients kyphosis Cobb's angle than treatment significantly improved P <0.05; observation group kyphosis Cobb's angle was significantly less than the control group, the difference was significant $\mathrm{P}<0.05$; 2-year follow-up observation group kyphosis Cobb's angle was significantly less than the control group, the difference was significant $\mathrm{P}$ $<0.05$; see Table 1 . 
Table 1 two groups of patients with kyphosis Cobb's angle comparison ( $\square \mathrm{x} \pm \mathrm{s}$ )

\begin{tabular}{lcccc}
\hline Group & Case & Before Treat & After Treat & After 2 years \\
\hline Observe Gr & 40 & $15.4 \pm 3.5$ & $8.8 \pm 0.6$ & $19.2 \pm 3.6$ \\
Competition Gr & 40 & $14.8 \pm 2.9$ & $11.3 \pm 1.4$ & $21.4 \pm 3.5$ \\
$\mathrm{t}$ & & 0.8349 & 10.3807 & 2.7712 \\
$\mathrm{p}$ & & 0.4063 & 0.0000 & 0.0070
\end{tabular}

After the observation group and the control group of patients pain patients before treatment were significantly alleviated, $\mathrm{P}<0.05$; pain relief observation group than the control group, the difference was significant $\mathrm{P}<0.05$.

\begin{tabular}{lccc}
\multicolumn{3}{l}{ Table 2 two groups of patients with back pain relief compared to the situation ( } & After Treat \\
\hline Group & Case & Before Treat & $\mathrm{X} \pm \mathrm{s}$ ) \\
\hline Observe Gr & 40 & $7.4 \pm 1.2$ & $0.8 \pm 0.2$ \\
Competition Gr & 40 & $7.2 \pm 1.4$ & $1.3 \pm 0.6$ \\
$\mathrm{t}$ & & 0.6860 & 5.0000 \\
$\mathrm{p}$ & 0.4948 & 0.0000
\end{tabular}

\section{Discussion}

Vertebral compression fractures are clinically spinal surgery in the more common fracture disease, nerve damage for thoracolumbar compression fracture patients need early surgery. However, for different treatment in the presence of the non-clinical nerve damage in patients with vertebral compression fractures, some scholars believe that the need for surgery as soon as possible to effectively avoid complications delayed nerve injury; in addition, some scholars maintain that without surgery, surgical treatment may not be effective to correct kyphosis or better to maintain the status of the correction. So no surgery cases idea was accepted by the majority.

The study of traditional Chinese medicine comprehensive treatment, in which patients by internal medicine at the same time, the portion of the spine pillow, making effective back muscle exercise and other Chinese medicine, relieve the injured lower back pain, while effective corrective intramuscular kyphosis, its treatment observation group than the control group. Chinese medicine believes that the fracture will hurt tendons, moving improper then aggravate bone loss, but excessive resting is accelerated muscle atrophy, excessive exercise is aggravating bone and joint injuries, too much to stay in bed and ignore the exercise is caused by back muscles acceleration weakened, or even cause it to disuse atrophy, so to follow the dynamic and static effective combination, tendons, bone and try to strengthen the principles of exercise.

In short, no nerve injury thoracolumbar vertebral compression fractures were treated with combined therapy of traditional Chinese medicine, can effectively relieve the injured speedy pain state is conducive to correct vertebral kyphosis and effective restoration of vertebral height, a significant effect.

\section{References}

[1] Yang Zengmin, Tong Xingye, Yang Jun. percutaneous vertebroplasty Reflection anesthesia complications [J]. Journal of Clinical Orthopaedics, 2015, 18 (6): 757-758.

[2] Cai Dongzhe, Meng Xiangqi, Hui Renghua. The techniques such as resetting adjustable external 
fixator without nerve injury thoracolumbar burst fractures[J]. Changchun University of Traditional Chinese Medicine, 2014, 30 (5): 937-940.

[3] Yu Yibo, Wushao Bin. Percutaneous vertebroplasty in the absence of nerve injury and obvious kyphosis osteoporotic thoracolumbar fracture nonunion Application[J]. Weifang Medical College, 2015, 37 (5 ): 347-348.

[4] Yang Kai, Wang Jun, Zhou Jiping. The limited osteotomy fixation of old fractures of thoracolumbar kyphosis [J]. Translational Medicine electronic journal, 2016,3 (2): 19-20.

[5] Yao Shujiang, Jiang Zewei, Yu Jianlin. a limited posterior osteotomy for treatment of old thoracolumbar fracture [J]. Journal of neck pain, 2015, 36 (4): 301-303. 